M. Ke11y, G.T. Wrixon, App1. Phys. Lett., 32(9), 525 1978).

W. Tsang, S.E. Schwarz, J. Appl. Phys. 50(5), 3459 1979).

. Godone, E. Bava, Int. J. of IR and MM waves, 2(6), 225 (1981).

.H. Rhoderick, Metal-semiconductor contacts, P. Hammond and D. Walsh Eds., Clarendon press, Oxford (1980)

.M. Sze, Physics of semiconductor devices, 2nd edition, Jiley-Interscience, New-York (1981).

1. Pyée, J.P. Pasquali, Proc. 5th Colloque OHD, Lille (France), (1979).

1. Pyée, Proc. 4th IEEE Int. Conf. IR and MM waves, Miami 1979), Conf. Digest Suppl. p. 17.

J.C. Bolomey, J. Cashman, S. E1 Habiby, M. Pyée, A. Comecon, J.C. Hénaux, J.J. Jimenez, R. Adde, G. Vernet, Proc th IEEE Int. Conf. IR and MM waves, Miami (1979), Conf. ig. p. 112; J.C. Bolomey, Ann. des Télécom. 34, 469 (1979). A. Sentz, M. Pyée, C. Gastaud, J. Auvray, J.P. Letur, Rev. Sci. Inst. 49(7), 926 (1978).

J.P. Auton, Appl. Opt. 6(6), 1023 (1967).

.L. Warner, in Millimetre and submillimetre waves, Chap. 2, F.A. Benson Ed., Iliffe Books Ltd., London (1969)

.E. Dickens, IEEE Trans. MTT15(2), 101 (1967).

. Kreisler, I. Dudouit, M. Pyée, Proc. 7th IEEE Int.Conf.

[R and MM waves, Marseille (1983), Conf. Digest p. 277, to be published.

3.J. Clifton, private communication.

C. Hénaux, G. Vernet, R. Adde, J.C. Bolomey, Int. J of IR and MM waves 4(3), 407 (1983).

C.M. Matarrese, K.M. Evenson, Appl. Phys. Lett. 17(1), 3(1970).

D.B. Rutledge, S.E. Schwarz, A.T. Adams, Infrared Phys. $18,713(1978)$

\section{ON THE IMPROVEMENT OF THE ELECTROMAGNETIC COUPLING BETWEEN INFRARED POINT-CONTACT DETECTORS AND LASER BEAMS*}

\section{A. Comerón**, J. J. Jiménez}

Institut d'Electronique Fondamentale (CNRS, LA 22) Université Paris-XI Bât 220, F-91405 Orsay, France

\section{and}

\section{J.-Ch. Bolomey}

Laboratoire de Signaux et Systèmes (CNRS-ESE)

Plateau du Moulon, F-91190 Gif-sur-Yvette, France

Received January 9, 1984

The electromagnetic coupling between a gausian beam and whisker structures such as those found in some kinds of non-1inear devices used at infrared frequencies is investigated. Calculations show the potential improvement of the electromagnetic coupling coefficient by the proper choice of the focusing angle of the beam and the use of corner reflectors, giving quantitative support to these techniques which have been used for long time.

Key words: infrared antennas, point-contact non-1 inear devices....

* Work supported in part by the CNRS, ATP 3547 and Greco Microondes.

** Present address: Escuela Técnica Superior de Ingenieros de Telecomunicación, Jorge Girona, s/n. Barcelona-34 Spain. 


\section{Introduction}

Infrared point-contact detectors and mixers can be considered as lumped junctions (Schottky, MIM or Josephson) supplied by means of a wire structure. The wire structure, the length of which is often much greater than the wavelength, acts as an antenna and provides a coupling to external beams $|1,2|$.

The detector performance (responsivity, sensitivity) is very dependent on this coupling. This paper presents some theoretical results concerning its optimization in the case of a whisker antenna irradiated by an electromagnetic gaussian beam. The antenna is assumed to be thin, lossless and long enough for supporting traveling wave currents. Actually such current distributions have been found to fit fairly well the antenna characteristics of long whisker structures in the submillimiter and infrared ranges $|2-4|$.

The assumption of gaussian 1 aser beams restricts the range of the focusing angles at which the interaction can be accurately calculated to the domain of validity of the paraxial approximation. Nevertheless we will see that for the case of long (relative to the wavelength) antennas, the domain of concerning focusing angles falls into the limits of the paraxial approximation.

We study the effects of two kinds of coupling improving devices: focusing mirrors or lenses and $90^{\circ}$ corner reflectors. We will deduce rules about the setting of these devices in order to have maximum power coupled by the antenna to the junction. We give calculated numerical values of the power available at the antenna terminals relative to the total power carried by the beam for some typical antenna lengths in the infrared range.
Electromagnetic coupling coefficient

The coupling strength between an antenna and an electromagnetic incident beam can be expressed by the value of the electromagnetic coupling coefficient. This coefficient is defined as the ratio

$$
\rho_{c}=\frac{W_{a}}{W_{i}}
$$

Wa is the available power at the antenna terminals and $W i$ is the total power carried by the incident beam. Optimizing coupling consists in making Wa as large as possible for a given Wi.

$\rho_{c}$ is related to the characteristics of the antenna and to the field distribution of the inci dent beam $|5|$ by the formula

$$
\rho_{c}=\frac{\left|\int \vec{F}(\hat{r}) \cdot \vec{E}_{+}(\hat{r}) d \Omega\right|^{2}}{\int|\vec{F}(\hat{r})|^{2} d \Omega \int\left|\vec{E}_{+}(\hat{r})\right|^{2} \mathrm{~d} \Omega}
$$

Here $\vec{F}(\hat{r})$ is the radiation vector of the antenna, proportional to the electric radiated field in emitting situation. $\vec{E}_{+}(\hat{r})$ represents the electric field distribution in the incoming beam far enough from the beam waist. $\hat{r}$ is the unit direction vector and $\mathrm{d} \Omega$ the elementary solid angle. The integrations are extended to all the directions. The coordinates origin is taken at the beam waist.

Schwarz's inequality shows that the absolute maximum of expression (2) is 1. For a given antenna (thus for a given $\vec{F}(\hat{r})$ ), optimizing coupling consists in creating a field distribution in such a manner that expression (2) becomes as near to l as possible.

Obviously, $\rho_{c}$ is equal to 1 when the following pattern and polarization matching condition is satisfied.

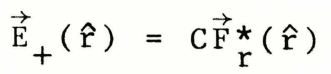


C being an arbitrary proportionality constant. However this condition may be difficult to achieve in the practice. A trade-off must be sought between the increase of $\rho_{c}$ and the complexity of the system used to implement the matching condition ( 3 ).

The radiation vectors of whisker structures have revolution symmetry. On the other hand, laser beams have a field fistribution concentrated around a given direction (Fig.1). So it is very difficult to manipulate this distribution to fit exactly the condition ( 3 ).

To calculate the numerical values of the electromagnetic coupling coefficient for different focusing conditions formula (2) has been computed $|6|^{*}$.

\section{Influence of focusing angle}

As it has been long known, the straightest manner of increasing coupling is to send the incident beam in the direction of the antenna ma ximum gain $|2|$. With regard to formula (2), that means overlapping the angular domains where $|\vec{F}(\hat{r})|$ and $\left|\vec{E}_{+}(\hat{r})\right|$ take their most significant values.

A simple way in which the field angular distribution of the laser can be modified is by using a focusing device: a lens or a sphericak mirror. The field distribution is varied by changing the focal length of the focusing device. This technique has proved experimentally to increase the power coupled to the non-linear device $|1,7|$. The coupling improvement arises from a better overlapping of $|\vec{F}(\hat{r})|$ and $|\vec{E}(\hat{r})|$.

* Though the calculations reported here concern a lossless, thin, traveling wave antenna, the structure of the program is general and it allows doing the calculations for any antenna radiation characteristic and for any field distribution in the incoming beam.

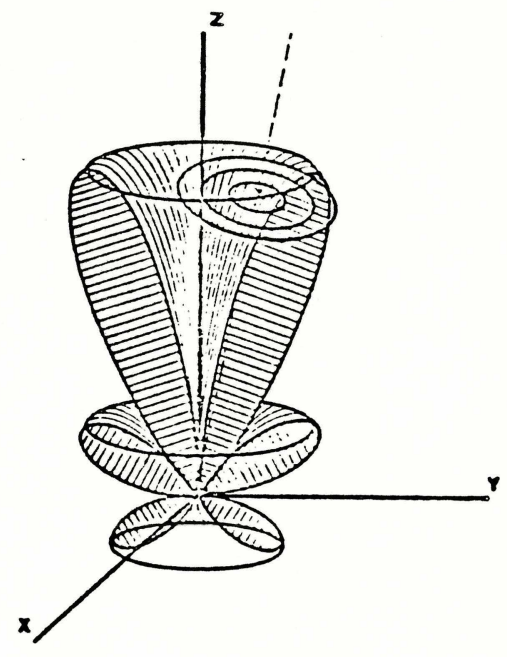

Figure l. Schematic representation of the radiation pattern from a cat-whisker antenna and of the field intensity in a laser beam focused upon it.

Our aim is to determine the focusing angle which maximizes the available power at the termi nals of the antenna.

The gaussian beam focusing angle $\Delta \theta_{i}$ is def $\underline{i}$ ned as the angle, measured from the beamwaist in a meridian plane over a spherical surface far enough from the waist, between points at which the beam intensity is $1 /$ e of its maximum value. We assume further that the beam is 1 inearly polarized, as when there are Brewster windows inside the 1 aser cavity. The variation of the electric field amplitude in the beam is proportional to

$$
e^{-\frac{2 \alpha^{2}}{\left(\Delta \theta_{i}\right)^{2}}}
$$

where $\alpha$ is the ingle with respect to the maximum intensity dire:tion (Fig. 2).

Figure 3 ; lows the results of calculations for three antenas, respectiveiy $50 \lambda, 25 \lambda$ and $10 \lambda$ long. These curves have been calculated 


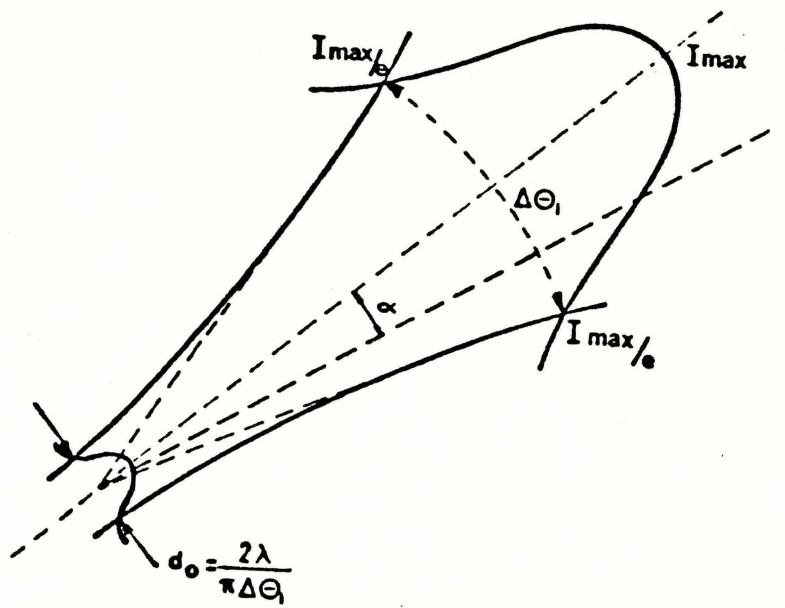

Figure 2. Gaussian beam. $I_{\max }$ is the maximum power intensity.

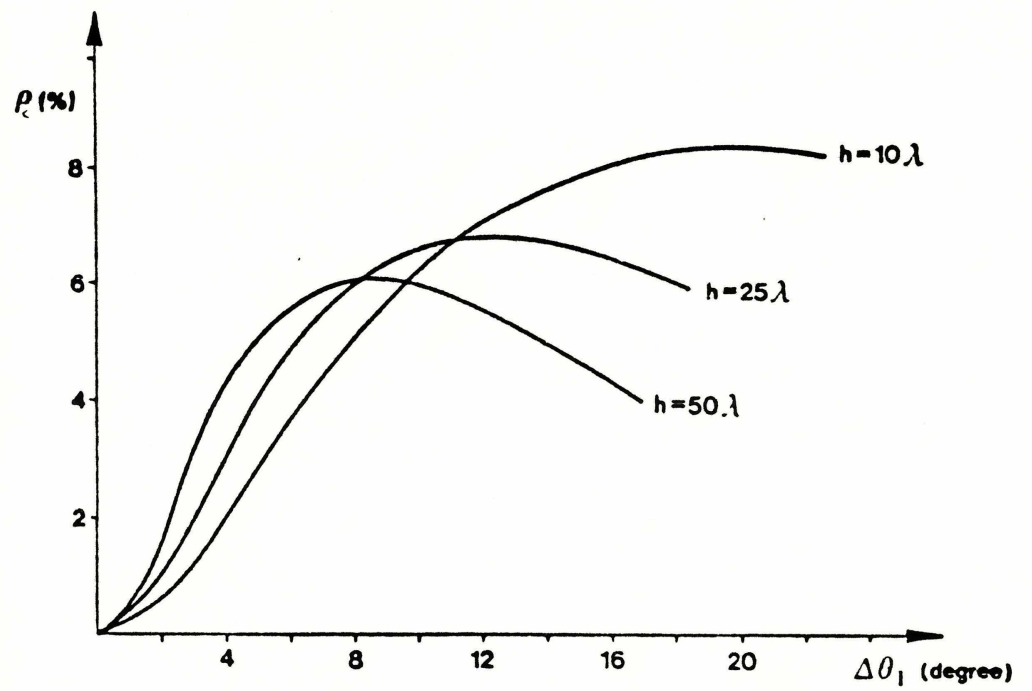

Figure 3. Variation of the electromagnetic coupling coefficient versus the focusing angle. The beam is assumed to be focused over the antenna terminal. assuming that the beam is sent along the direction of the antenna maximum gain and that it is focused at the antenna terminal. From Fig. 3 we can draw the following remarks: a) there exists a focusing angle which maximizes the coupling coefficient; b) at small focusing angles the coupling coefficient is higher for the longer antennas. This is due to the fact that longer antennas are more directive than the shorter ones and for small focusing angles we approach the condition of an incident plane wave; c)the maximum coupling coeffi cient is higher for the shorter antennas. This results from the overlapping of $|\vec{F}(\hat{r})|$ and $|\vec{E}(\hat{r})|$ in formula (2) over a wider angular range; d) teven at the angles which maximize $\rho_{c}$, this coefficient takes values which are far from the unit limit.

The coupling coefficient may be still improved by a proper choice of the focusing point.

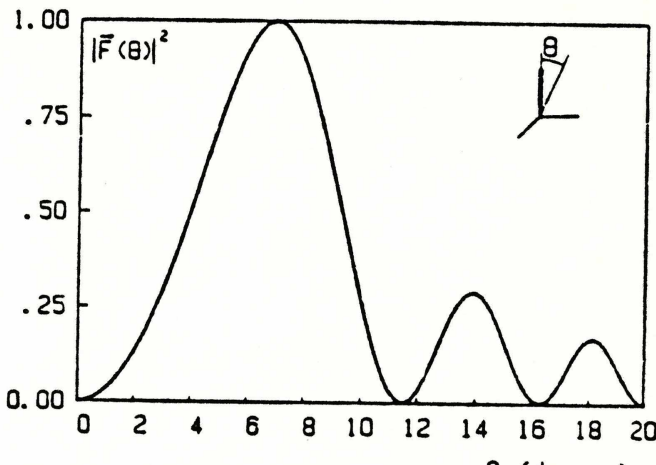

$\theta$ (degree)

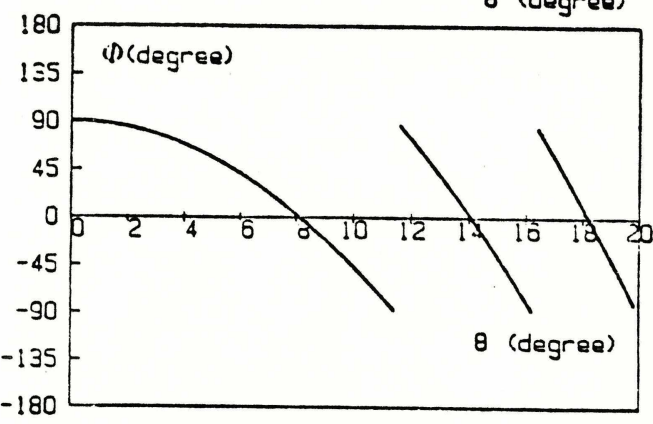

Figure 4. Calculated magnitude (in arbitrary units) and phase of the radiation vector for a $50 \lambda$ traveling - wave antenna. Origin taken at the antenna terminal. 
In Fig. 4 we have represented the magnitude and phase variations of $\vec{F}(\hat{r})$ in a meridian plane for a $50 \lambda$ traveling wave antenna when the coordinates origin is taken at the antenna terminal (the axis of the spherical coordinates system is taken in the same direction as the antenna axis).

We see that the phase undergoes a $180^{\circ}$ shift over each lobe. Furthermore, the phase shift is approximately $-90^{\circ}$ in the maximum radiation direc tion $\theta_{M}$. Previously we stated that in formula (2) the coordinates origin has to be taken at the beam waist. Thus Fig. 4 gives the phase variations of the radiation vector which must be used when the incident beam is focused at the antenna terminal. The magnitude of the radiation vector does not depend on the origen location. However the phase variations are affected by it.

If the antenna is translated along its axis for a distance $z_{o}$, the radiation vector must be mu1tiplied by exp $\left[\mathrm{j} \phi\left(z_{0}, \theta\right)\right]$, with

$$
\phi\left(z_{0}, \theta\right)=-\frac{2 \pi z_{o}}{\lambda} \cos \theta
$$

$\lambda$ being the beam wavelength.

This phase shift can be used to compensate for the original phase variations shown in Fig. 4 Choosing $z_{0}$ in such a manner that $\phi\left(z_{0}, \theta_{M}\right)$ equals +900 one obtains $z_{0}=h / 2, h$ being the antenna length. For derivating this simple result the crude approximate value $\theta_{M} \simeq \sqrt{\lambda / h}$, valid for long antennas, has been used.

Fig. 5 shows the phase variations when the beam is focused at the middle of the antenna. In this case the most significant contributions to the integral in the numerator of expression (22) add in phase. Thus, an improvement of the maximum cou pling coefficient must be expected. Though Fig.5 has been calculated for a $50 \lambda$ antenna, similar phase variations are found for traveling wave antennas of different lengths, provided that the

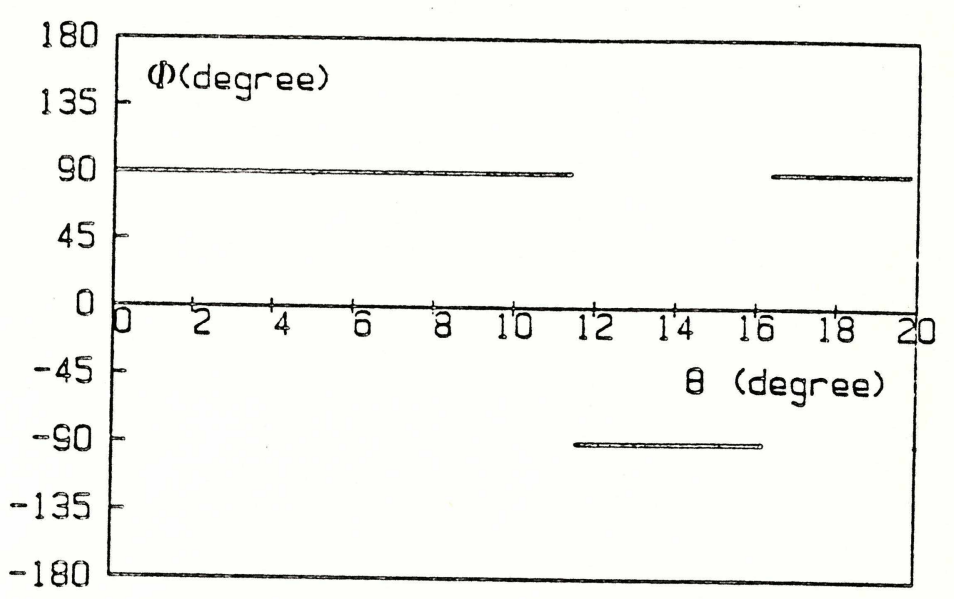

Figure 5. Calculated phase variation of the radiation vector for a $50 \lambda$ traveling-wave antenna. Origin taken over the antenna axis at $25 \lambda$ from the terminal.

origin is taken at half the length.

In Fig. 6 it has been as sumed that the beams are focused at the antennas half length. The basic features of the curves are the same as in Fig. 3. Nevertheless the maxima are sharper, they are shif ted to smaller values of $\Delta \theta_{i}$ and, as expected, are higher than those in Fig. 3 .

Fig. 6 shows in addition that, at the focusing angle which maximizes $\rho_{c}$ for each antenna, the projection of the antenna over the beam waist plane equals approximately the waist diameter as defi ned by the expression (see Fig. 2).

$$
\mathrm{d}_{\mathrm{o}}=\frac{2 \lambda}{\pi \Delta \theta_{i}}
$$

This corresponds to the intuitive condition of the beam power being distributed in an uniform way over the an tenna length. This fact can be used to give an analytical expression for the optimum focusing angle. From Fig. 7 it can be seen that this angle corresponds to the condition 


$$
\mathrm{h} \sin \theta_{M}=\mathrm{d}_{\mathrm{O}}
$$

By substitution of (5) in (6) we find

$$
\Delta \theta_{i}=\frac{2 \lambda}{\pi h \sin \theta_{M}}
$$

For traveling wave antennas the angle between the antenna axis and the maximum gain direction is given by $|2,8|$

$$
\theta_{M}=\operatorname{arcos}\left(1-0.371 \frac{\lambda}{h}\right)
$$

This expression can be substituted in (6) to find the value of $\Delta \theta_{i}$ which maximizes the coupling coefficient. A specially simple expression can be obtained for antennas of length about $4 \lambda$ or 1 onger. In the practice such long antennas are common at infrared frequencies. This is due to the fact that etching techniques $|6|$ used to make tips do not permit obtaining whiskers shorter than about $200 \mu \mathrm{m}$. Actually, for these long antennas, expression (8) can be simplified to give $|5,6|$

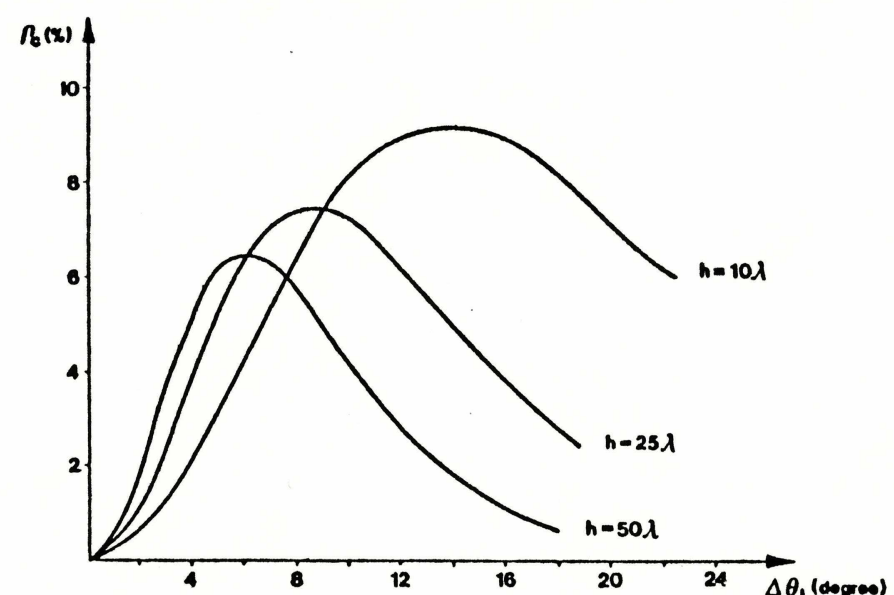

Figure 6. Variation of the electromagnetic coupling coefficient versus the focusing angle. The beam is assumed to be focused at the antenna half length.

$$
\theta_{M}=0.861 \sqrt{\frac{\lambda}{h}}
$$

In the formula (7), sin $\theta_{M}$ can be substituted by the expression (9) as the angle is small enough, thus obtaining

$$
\Delta \theta_{i}=0.74 \sqrt{\frac{\lambda}{4}}
$$

It is to be noted that the expression of $\Delta \theta$. given by (10) is not very different from that of $\theta_{M}$ given by (9) which, in turns, for these long antennas, gives also the approximative value of the main lobe width in a meridian plane.

The rules giving the resultant focusing angle for a given beam and a given lens or mirror can be found in reference 9 . It must be taken into account that in reference $\exists$ beam widths are defined between points of l/e of the maximum field, whilst we have defined them between points of $1 / \mathrm{e}$ of the maximum intensity (Fig. 2).

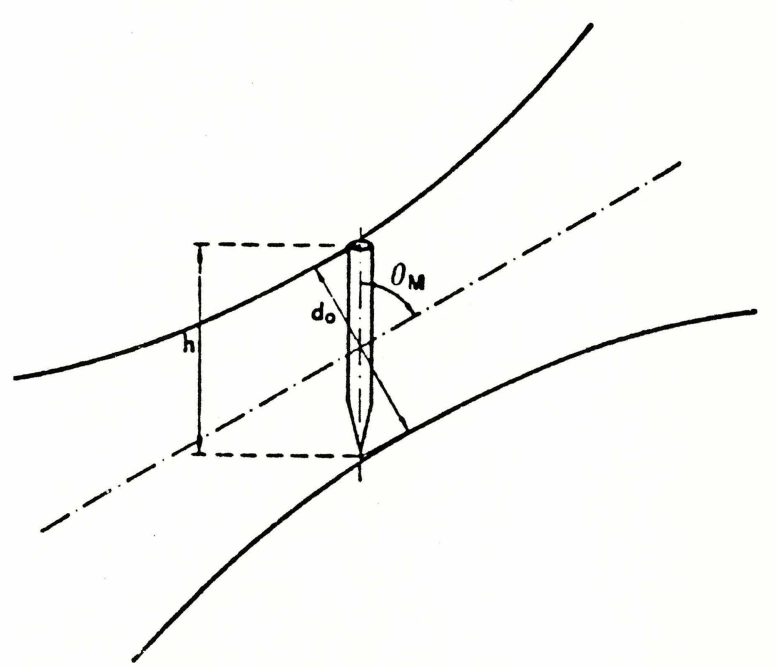

Figure" Relation between the antenna length ald the beam waist at the optimal focusing angle. 


\section{$90^{\circ}$ corner reflectors}

We have seen that by a proper adjustment of the focusing angle the coupling coefficient can be improved. Still, we fall very far from the total coupling expressed by condition ( 3 ).

A more efficient manner of matching the $\vec{F}(\hat{r})$ and $\vec{E}_{+}(\hat{r})$ distributions consists in trying to match the radiation vector to the incident field pattern.

This has been done by placing reflectors in the neighborhood of the antenna |10-12|. The combination of the antenna and the reflector can be thought as a new antenna which has no longer rotational symmetry ( $\vec{F}(\hat{r})$ is now a function of $\theta$ and $\phi)$ and its radiation is concentrated in a more reduced domain of directions. In this way, though it is still difficult to satisfy the condition (3) for every direction, the overlapping may be more complete. The distance between the reflector and the antenna must be chosen in order to reduce the secondary lobes which would contribute to decrease the antenna gain.

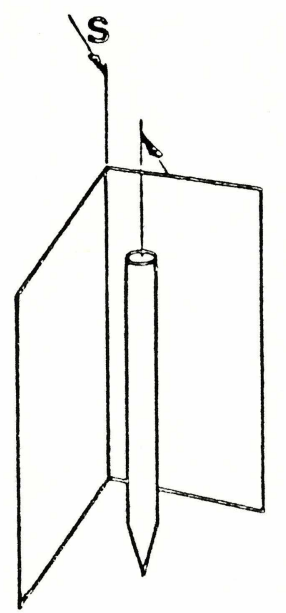

Figure 8. Whisker antenna . with a $90^{\circ}$ corner reflector.
Reflectors and focusing devices can be used together in order to obtain the best coupling coefficient.

We present here the computation results for antennas in the proximity of a $90^{\circ}$ corner reflec tor (Fig. 8) with the antenna axis contained in the bisector plane of the corner. In order to find the resulting antenna patterns we apply the images method. The equivalent of Fig. 8, if the corner is assumed to be made of a perfect conduc tor, is shown in Fig. 9, where the arrows indicate the relative senses of current in the antenna and its images. Kräutle at al |10| have studied the optimal spacing between a thin traveling-wave antenna and a 900 corner reflector for an antenna length of $4 \lambda$. They have found that the distance between the antenna and the corner which maximizes the antenna gain is about 1.2 $\lambda$. In Fig. 10 we have plotted the calculated squared magnitude of the radiation vector in the bisector plane versus the angle with respect to the antenna axis for a $4 \lambda$ antenna and a $1.2 \lambda$ spacing. It can be seen that secondary radiation lobes have virtually disappeared.

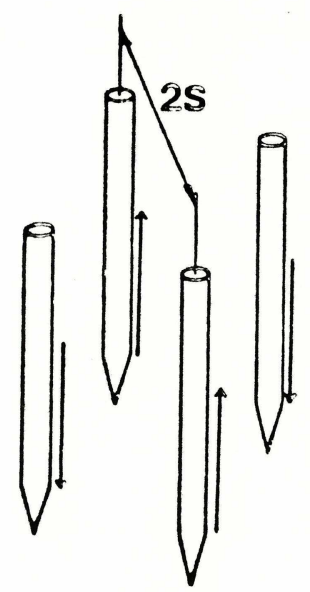

Figure 9. Image equivalent of $\mathrm{Fiz}_{\mathrm{z}} \mathrm{8}$ 


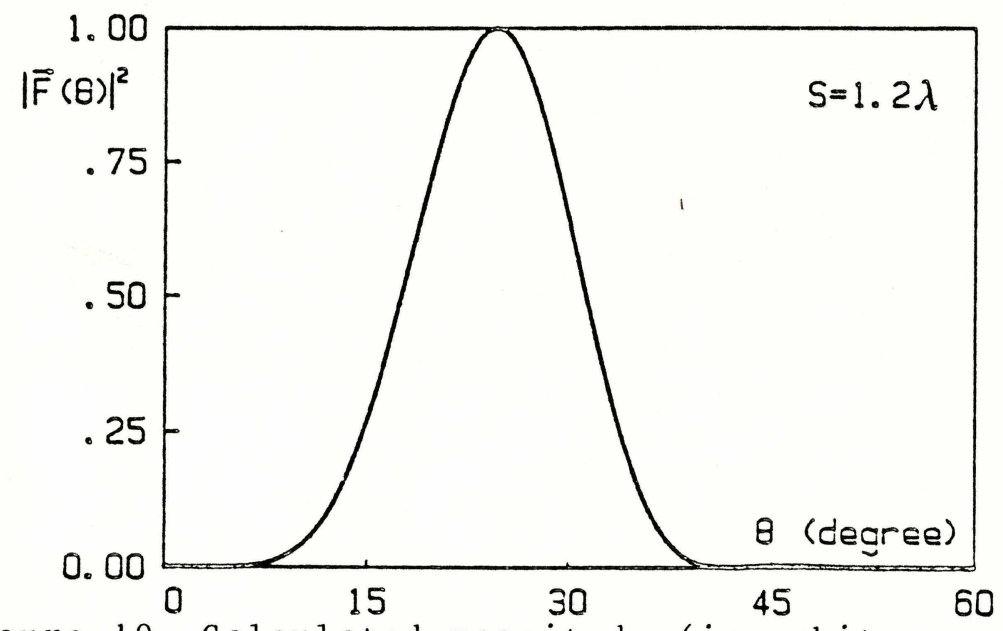

Figure 10. Calculated magnitude (in arbitrary units) of the radiation vector in the corner bisector plane for a $4 \lambda$ antenna and a $1.2 \lambda$ spacing.

A case of more practical interest at higher infrared frequencies is that of a $25 \lambda$ antenna. Fig. 11 shows patterns analog to that of Fig. 10 The decrease of secondary lobes is more important for spacings between $2.5 \lambda$ and $3 \lambda$. Fig. 12 is ana$10 g$ to Fig. 11 for a $50 \lambda$ antenna. It can be seen that the secondary lobes begin to weaken at spacings around $3.2 \lambda$. Furthermore the range of spacings where the weakening is appreciable is not very narrow. Comparing the plots for optimum spacing (the spacing which makes the secondary lobes to vanish) with formulae (8) or (9) it can be seen that the direction of maximum gain in the bisector plane of the corner occurs at about the same zeni thal angle as for the antenna without reflector.

It is to be noted that the calculated optimal spacings agree fairly well with what can be predicted from the following statement: the opti mal spacing occurs when the contributions to radiation by the antenna and its images add in phase in the direction corresponding to the anten na maximum gain $\theta_{M}$. With the current senses indicated in Fig. 9 this occurs in the bisector plane of the corner for a spacing $S$ which satisfies
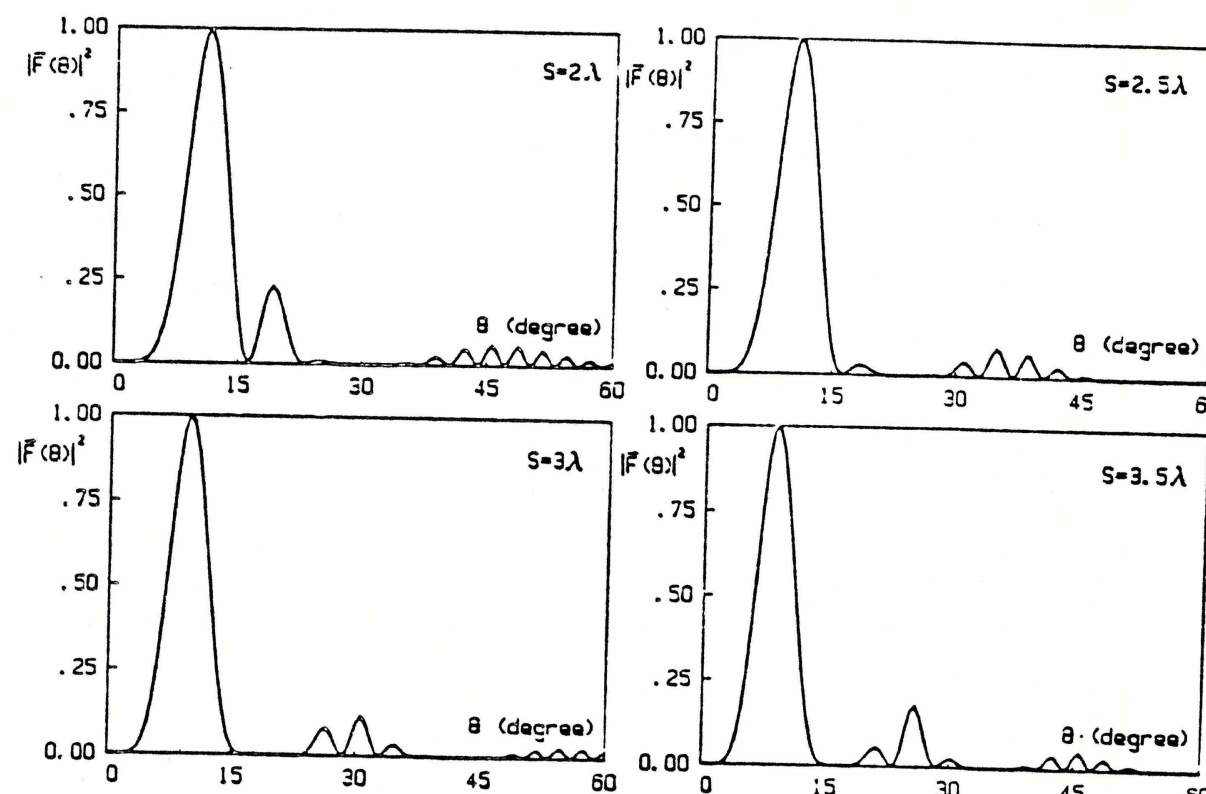

.

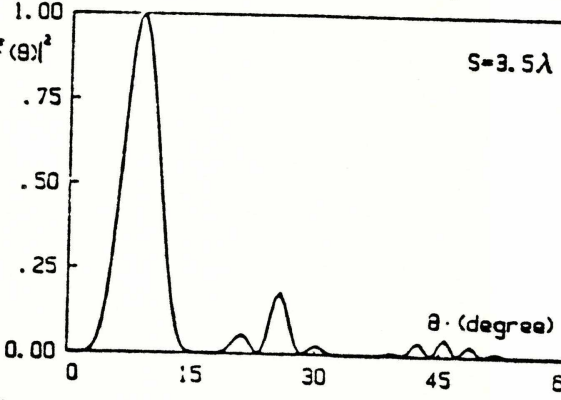

Figure 11. Calculated magnitude (in arbitrary units) of the radiation vector in the corner bisector plane for a $25 \lambda$ antenna and several spacings.

the condition

$$
S \sin \theta_{M}=\frac{\lambda}{2}
$$

(see Fig. 13).

If the antenna is long enough, formula (9) stands and (11) becomes.

$$
-\frac{S}{\lambda}=\frac{1}{2 \times 0.861} \sqrt{\frac{h}{\lambda}}
$$

Expression ( 12 ) gives the optimal spacing-towavelength ratio as function of the antenna lengthto-wavelength ratio. The optimal spacing is proportional to $\sqrt{\lambda}$, which is an attractive feature for using corner reflectors at short wavelengths. 

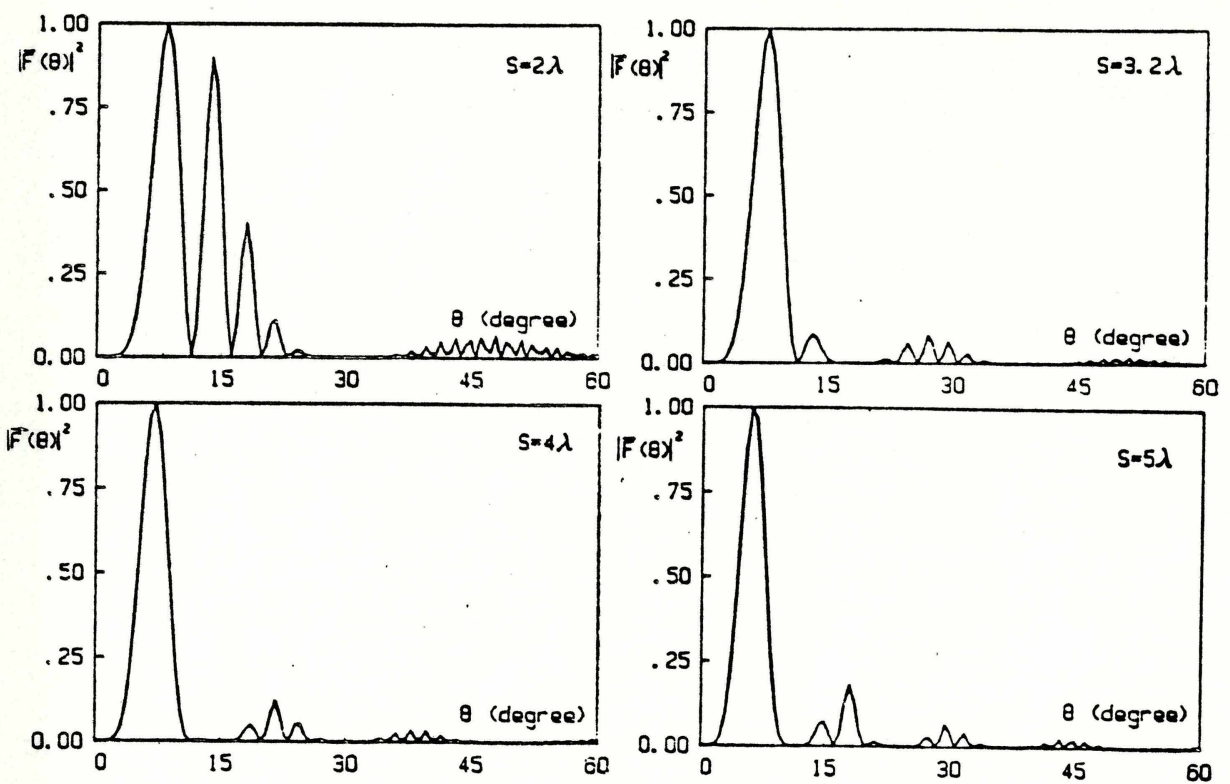

Figure 12. Calculated magnitude (in arbitrary units) of the radiation vector in the corner bisector plane for a $50 \lambda$ antenna and several spacings.

The relative $1 \mathrm{ack}$ of sensitivity of the antenna gain to spacing variations for long antennas can be qualitatively explained regarding to Fig. 13 . If $S$ satisfies the condition (11), the contributions of all the radiators add in phase in the direction of $\theta_{M}$. However, if $\theta_{M}$ is sma11, a slight difference in the value of $S$ will originate only small phase shifts in the adding contributions.

In Fig. 14 we have represented the calculated evolution of the coupling coefficient with the focusing angle for the $50 \lambda$ antenna at several spacings near to the optimum. The beam is assumed to propagate along the direction of maximum gain in the corner bisector plane. The plotted results have been calculated for a beam waist lying over the intersection of the plates which constitute the reflector, at equal distance of both antenna ends. The phase of the radiation vector is almost constant over each lobe when the origin is taken

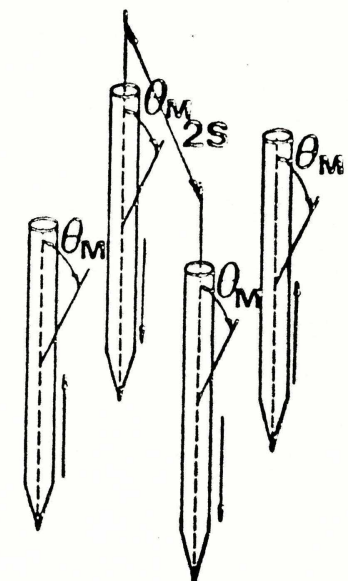

Figure 13. Determination of the optimal spacing.

at that point (a condition similar to that repre sented in Fig. 5). The curve corresponding to the antenna without the reflector in Fig. 14 is that of the $50 \lambda$ antenna in Fig. 6. The logarithmic vertical scale in Fig. 14 has been used in order to ease the comparison between the curves. The 0 level corresponds to a coupling coefficient of 1 .

Fig. 14 shows that:

a) The coupling coefficient is strongly increased by an optimally spaced reflector (more than $6 \mathrm{~dB}$ for all focusing angles, $9 \mathrm{~dB}$ for optimal angle).

b) If the proper focusing angle is used, the elec tromagnetic coupling coefficient can reach values around 0.4 , i.e. $40 \%$ of the power carried by the beam would be available from the antenna.

c) The calculation confirms, as Fig. 12 and 14 suggested, that the spacing is not very criti cal in order o obtain an appreciable increase in the coilpling coefficient for a long-wire antenna. 


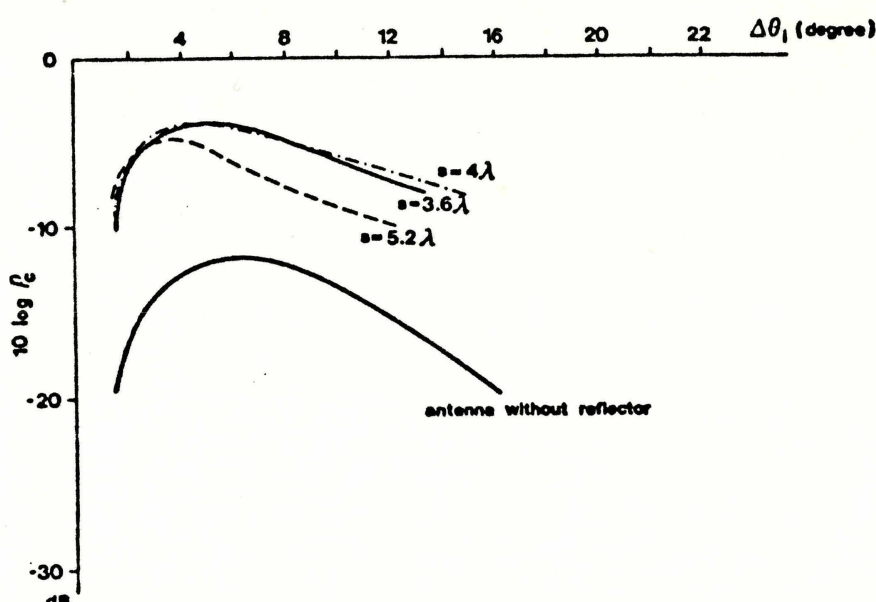

Figure 14. Calculated electromagnetic coupling coefficient versus focusing angle for a $50 \lambda$ antenna at several spacings from a $90^{\circ}$ corner reflector.

\section{Conclusions}

We have studied numerically the electromagne tic coupling between a thin traveling-wave antenna and a gaussian electromagnetic beam. This is of practical interest in the improvement of performances of non-linear point-contact devices (MIM junctions, Schottky diodes....) in the submillimeter and infrared ranges.

We have presented two techniques for increasing the electromagnetic coupling.Both of them have been used before $|1,7,10-12|$ and proved to be useful. We have shown that these techniques are theoretically justified by formula (2), which indicates the importante of matching the antenna radiation vector and the electric field distribution of the incoming beam in order to in crease the electromagnetic coupling coefficient.

The matching can be improved by a proper mo dification of the incident beam characteristics. The simplest way of achieving this is by acting on the beam focusing angle. Our calculations show that it exists, for a given antenna, a focusing angle which maximizes the coupling coefficient. We have given an approximative expression for this angle. The maximum is higher for the shorter (in terms of wavelength) antennas. However, when choosing the antenna length it should be taken into account that the antenna impedance depends on its length. Thus a trade-off should be sought between the increase of the electromagnetic coupling coefficient and an eventual mismatch between the antenna and its load. Anyway for the long antennas used in the infrared range the coupling coefficient is not higher than $10 \%$ even for the best focusing conditions. Nevertheless it should be noted that, in addition to the simplicity of the technique, it may represent a significant increase relative to the coupling obtained with an improper focusing or no focusing at all.

Another way of improving the matching between the radiation vector and the incident field consists in modifying properly the former. This can be achie ved by placing reflectors in the vicinity of the antenna. An expression giving the optimal spacing between a $90^{\circ}$ corner reflector and a long antenna has been presented. Numerical calculations confirm its validity. The calculated numerical values of the electromagnetic coupling coefficient show that $f$ or antenna lenghts ranging from $25 \lambda$ to $50 \lambda$ the available power at the antenna terminals may be about $40 \%$ of the total power carried by the beam for properly chosen focusing angles.

Calculations show in addition that the accura cy in the spacing is not very critical for long antennas, a feature which diminishes the difficulty of using $90^{\circ}$ corner reflectors at short wave lengths.

Though not considered here, the variation of the antenna impedance by the presence of the reflectors should again be taken into account when optimizing the power transfer to the non-linear load. 


\section{$\underline{\text { References }}$}

1 1 V. Daneu, D. Sokoloff, A. Sänchez, and A. Javan, App 1. Phys. Lett. 15, 398 (1969).

|2| L.M. Matarrese, and K.M. Evenson, App1. Phys . Lett. $17,8(1970)$

|3| J.-Ch. Bolomey, and S. E1 Habiby, Int'1. Jour. IR \& MM Waves $\underline{2}, 541$ (1981).

|4| J.-Ch. Bolomey, J. Cashman, S. E1 Habiby, and D. Lesse1ier, Int'1. Jour. IR \& MM Waves $\underline{2}, 859$ (1981).

|5| J.-Ch. Bolomey, Annales des Télécommunications 34, $469(1979)$.

6 6 A. Comerón, "Contribution à 1'étude des diodes Méta1-Isolant-Métal (MIM) et Méta1-Vide-Méta1 (MVM). Couplage électromagnétique d'un Faisceau laser avec une diode à pointe". Docteur-Ingénieur Thesis, Université Paris-Sud, Orsay (France) (1980).

|7| L.0. Hocker, D.R. Sokoloff, V. Daneu, A.Szöke, and A. Javan, Appl. Phys. Lett. 12, 401 (1968).

$|8|$ H. Jasik, Editor, "Antenna Engineering Handbook", Mc Graw-Hil1 Book Company, New York 1961, Chap ter IV.

|9| H. Koge1nik, and T. Li, Proc. IEEE 54, 1312 $(1966)$.

|1 $0 \mid$ H. Kräutle, E.Sauter, and G.V. Schultz, Infrared Phys. 17, $477(1977)$.

|11| H. Kräut1e, E.Sauter, and G.V. Schultz, Infrared Phys. 18, 705 (1978).

$12 \mid$ H.R. Fetterman, P.E. Tannenwald, B.J. Clifton, C.D. Parker, W.D. Fitgerald, and N.R. Erickson, App 1. Phys. Lett 33, 151 (1978).

\section{CONFERENCE ANNOUNCEMENT}

\section{Ninth Annual International Conference on Infrared and Millimeter Waves}

Takarazuka City, Japan, October 22-26, 1984

\section{TRAVEL PACKAGE FOR NORTH AMERICANS}

In an effort to ameliorate the high cost of travel to Japan in October for our delegates, a travel package has been assembled. It is necessary for each delegate to make his arrangements with

Commerce Tours International, Inc.

870 Market Street

San Francisco, CA 94102

Tel: (415) 433-3072

Note 非: The Takarazuka Hotel is rather small (90 rooms). It is necessary to make your arrangements early if you wish to stay there. The Commerce Tours agent will book you into a first class hotel in Osaka for a reasonable price and will arrange a bus for our delegates to go to the Conference. Those staying in Osaka may find a wider variety of activities in the evening.

Note 非: Please be sure to tell your Commerce Tours agent what you want to do after the conference if you do not want to depart for the USA on October 26 th at 3 P.M. If you wish to stay in Osaka or visit another city, your agent will make all of the arrangements and will book your return to the USA later at your convenience. 